\title{
Jens Weidmanns Mahnungen sollten vernehmbar bleiben
}

Im Oktober verkündete Jens Weidmann überraschend seinen Rücktritt als Bundesbankpräsident zu Ende 2021. Das ist zu bedauern, da ein sehr guter, kritisch denkender und sympathischer Ökonom die Bundesbank verlässt. Aber eine „Katastrophe für unser Geld", wie die Bild-Zeitung schreibt, ist der Rücktritt nicht. Jens Weidmann ist seit zehn Jahren Bundesbankpräsident und damit auch Mitglied im Rat der Europäischen Zentralbank (EZB), also dem Gremium, das die geldpolitischen Entscheidungen trifft. In diesen zehn Jahren war die EZB im permanenten Krisenmodus: die Nachwehen der Finanzkrise, die Staatsschuldenkrise, Deflationsrisiken, die CoronaPandemie. In dieser Zeit hat die EZB neue Aufgaben übernommen, neue Instrumente eingesetzt. Die Geldpolitik war und ist dabei extrem expansiv ausgerichtet.

Jens Weidmann hat immer wieder auch auf die Risiken hingewiesen, die mit den neuen Aufgaben, den neuen Instrumenten, der extrem expansiv ausgerichteten Geldpolitik verbunden sind. Er hat insbesondere darauf gepocht, geld- und fiskalpolitische Aufgaben strikt voneinander zu trennen. Warum ist das eigentlich so wichtig? Die EZB ist eine unabhängige Institution, d. h., sie unterliegt nicht der direkten parlamentarischen Kontrolle. Begründet wird die Unabhängigkeit mit der Reduzierung von Inflationsrisiken. Politiker:innen, die sich Wahlen stellen müssen, haben einen Anreiz, kurzfristige Wahlerfolge durch eine mit Inflation verbundene Geldpolitik zu erlangen. Der Verzicht auf die direkte parlamentarische Kontrolle eines Politikbereichs kann trotzdem nur unter ganz bestimmten Voraussetzungen gerechtfertigt werden; unter anderem dürfen mit dieser Politik keine signifikanten Verteilungseffekte verbunden sein und keine hoheitlichen Aufgaben wahrgenommen werden. Diese Voraussetzungen sind bei der Geldpolitik grundsätzlich gegeben, bei der Fiskalpolitik jedoch genau nicht. Fiskalpolitik ist immer mit erheblichen Umverteilungseffekten verbunden. Fiskalpolitische Entscheidungen müssen demnach demokratisch legitimiert werden! Deshalb ist die strikte Trennung zwischen einer unabhängigen Geldpolitik und einer der parlamentarischen Kontrolle unterliegenden Fiskalpolitik von überragender Bedeutung.

Jens Weidmann hat immer wieder vor einem Verschwimmen von Geld- und Fiskalpolitik gewarnt. Sei es nach der Finanzkrise, als die EZB den Banken Notfallkredite zur Verfügung stellte oder als die Bankenaufsicht auf die EZB übertragen wurde oder als im Zuge der Staatsschuldenkrise das Instrument der OMT (Outright Monetary Transactions) eingeführt wurde. Dieses Instrument erlaubt es der EZB, in Krisensituationen unter bestimmten Bedingungen, Staatsschulden von Mitgliedsländern in finanziellen Schwierigkeiten zu kaufen, mit dem Ziel, destabilisierende Wirkungen auf die Währungsunion als Ganzes zu begrenzen. 2014 waren die Inflationsraten im Euroraum sehr niedrig (sie lagen teilweise im negativen Bereich), das Wirtschaftswachstum war schwach, die Arbeitslosenquote hoch, die Kreditvergabe der Banken rückläufig. Das gesamtwirtschaftliche Umfeld verlangte also nach einer expansiven Geldpolitik. Aber die EZB hatte mit ihren herkömmlichen Instrumenten bereits die effektive Zinsuntergrenze erreicht. So beschloss der EZB-Rat, das Instrument des Quantitative Easing einzusetzen und in großem Umfang vor allem Staatsanleihen zu kaufen. Ziel dieser Ankäufe ist es, die langfristigen Zinsen direkt zu senken, um somit Konsum und Investitionen und damit die gesamtwirtschaftliche Nachfrage anzukurbeln, um Preise und Wirt-

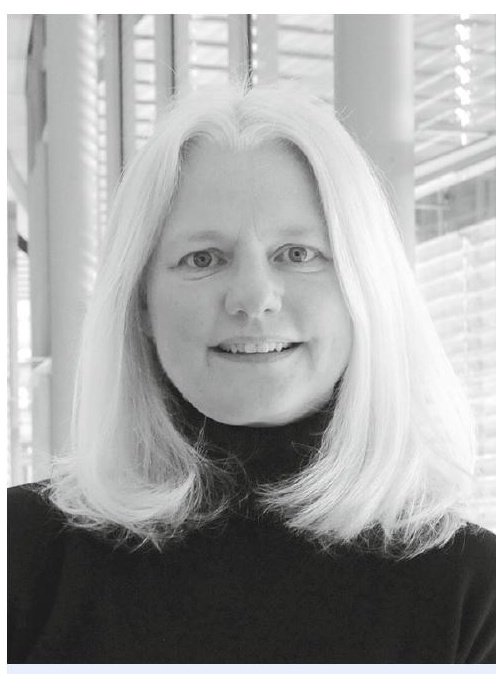

Prof. Dr. Ulrike Neyer ist Professorin für Volkswirtschaftslehre, insbesondere Monetäre Ökonomik an der HeinrichHeine-Universität Düsseldorf. 
schaftswachstum zu stimulieren. Auch hier hat Jens Weidmann insbesondere die Nähe zur Fiskalpolitik problematisiert. In Reaktion auf den Ausbruch der Corona-Pandemie hat die EZB das PEPP (Pandemic Emergency Purchase Programme) aufgelegt. Dieses Programm erlaubt der EZB, in großem Umfang Wertpapiere wie Staats-, aber auch Unternehmensanleihen zu kaufen. Ziel dieses Programms war es erstens, die Finanzmärkte zu stabilisieren, und zweitens, günstige Finanzierungsbedingungen in der Pandemie zu schaffen bzw. aufrechtzuerhalten. Hier hat Jens Weidmann darauf gedrungen, dass das Programm zeitlich begrenzt und eindeutig an die Krise gebunden sein muss. Das „P“ stünde eben nicht für „Permanent“, sondern für „Pandemic“. Jens Weidmann hat auch allgemein auf die Risiken hingewiesen, die mit dem Ankauf von Staatsanleihen verbunden sind, wie die Schwächung der Disziplinierung durch die Finanzmärkte und damit des Anreizes zu solidem Staatshaushalten. Auch die Problematik der fiskalischen und finanziellen Dominanz, die durch eine extrem expansive Geldpolitik über einen langen Zeitraum verstärkt wird, hat er betont. Die De-facto-Unabhängigkeit der EZB ist damit in Gefahr, da sie in das Schlepptau der Fiskalpolitik und der Finanzmärkte gerät.

Die Risiken sind die eine Seite der Geldpolitik der vergangenen Jahre. Auf der anderen Seite hat die Geldpolitik in den Krisen aber auch entscheidend zur Stabilisierung des Finanzsystems, der Währungsunion, der Preise und der wirtschaftlichen Entwicklung im Euroraum beigetragen: Der Bankensektor ist nicht zusammengebrochen, die Währungsunion nicht auseinandergebrochen, die Finanzmärkte sind in der Pandemie nicht zum Erliegen gekommen. Die EZB hat also erfolgreich als Lender of Last Resort für Banken, Staaten und Märkte agiert. Auch wiesen seit 2015 bis zum Ausbruch der Pandemie die Preise und die wirtschaftliche Entwicklung im Euroraum einen positiven Verlauf auf.

Die stabilisierenden Wirkungen der extrem expansiv ausgerichteten Geldpolitik auf der einen und die Risiken auf der anderen Seite wurden und werden im EZB-Rat unterschiedlich eingeschätzt und gewichtet. Jens Weidmann hat insbesondere den Risiken ein sehr hohes Gewicht beigemessen. Mit seinem Ausscheiden aus dem EZB-Rat wird diese kritische, mahnende Stimme fehlen. Eine „Katastrophe für unser Geld“ ist das jedoch nicht. Die Stabilität des Geldes hängt nicht von einer einzelnen Person ab. Geldpolitische Entscheidungen werden von den jeweils 19 stimmberechtigten Mitgliedern des 25-köpfigen EZB-Rats gefällt - innerhalb des innen durch die EU-Verträge gesteckten gesetzlichen Rahmens. Für die Stabilität des Geldes spielen also die EU-Verträge und der gesamte 25-köpfige EZB-Rat eine entscheidende Rolle. Das Ausscheiden eines einzelnen aus diesem Rat kann also nicht mit einer Katastrophe gleichgesetzt werden - genau das wäre eine Katastrophe! Dieses wäre ein Indiz für einen total mangelhaften gesetzlichen Rahmen und für in der Mehrheit inkompetente Ratsmitglieder!

Der Einfluss des einzelnen Ratsmitglieds besteht also zum einen in seinem Abstimmungsverhalten und zum anderen in seiner Überzeugungskraft in der Diskussion im Rat. Für Entscheidungen, denen das gründliche Abwägen von Chancen und Risiken zugrunde liegt, wobei die Chancen und Risiken wiederum von komplexen, unsicheren wirtschaftlichen Zusammenhängen bestimmt werden, ist es wichtig, dass diese von einem Gremium getroffen werden, deren Mitglieder a) die Fachkompetenz haben, aber auch unterschiedliche Sichtweisen mitbringen, b) die Diskussion unterschiedlicher Sichtweisen zielführend erlauben und c) Entscheidungen gut in der Öffentlichkeit kommunizieren und erklären können. Letzteres ist für die Zentralbank wichtig, da dieses Vertrauen in die Institution Zentralbank schafft, eine unabdingbare Voraussetzung für eine erfolgreiche Geldpolitik. Die Nachfolge von Jens Weidmann sollte a) die fachliche Kompetenz haben und auch den Risiken der derzeitigen geldpolitischen Ausrichtung eine Stimme geben, damit die Diversität der Meinungen im EZB-Rat erhalten bleibt, b) Argumente überzeugend und kompetent darlegen können und c) im Diskurs getroffene Entscheidungen gut in der Öffentlichkeit kommunizieren können. 